\title{
Guest editorial: Advances in tools, techniques and practices for multimedia $\mathrm{QoE}$
}

\author{
Shelley Buchinger • Rui J. Lopes • \\ Satu Jumisko-Pyykkö • Hans-Jürgen Zepernick
}

Published online: 29 August 2014

(C) Springer Science+Business Media New York 2014

It has been realized that the success of multimedia services or applications relies on the analysis of the entire user experience (UX). The relevance of this paradigm ranges from Internet protocol television to video-on-demand systems for distributing and sharing professional television (TV) and user-generated content that is consumed and produced ubiquitously. To obtain a pleasurable user experience, a large amount of aspects have to be taken into account. Major challenges in this context include the identification of relevant UX factors and the quantification of their influence on Quality of Experience (QoE). This special issue is dedicated to advances in, tools, techniques and practices for multimedia QoE that tackle several of the aforementioned challenges. It consists of six papers that address three of these topics: advanced metrics for QoE, application instrumentation for QoE assessment, and integration of QoE aspects in the complete networked multimedia production-consumption chain. The special issue's papers are briefly described below.

The first topic is addressed in the papers by Barkowsky et al. and Eg et al. The paper entitled "Hybrid video quality prediction: Reviewing video quality measurement for widening

\footnotetext{
S. Buchinger $(\varangle)$

Cognitive Science Research Platform, University of Vienna, Wien, Austria

e-mail: shelley.buchinger@univie.ac.at
}

R. J. Lopes

Instituto de Telecomunicações (IT-IUL), Lisboa, Portugal

e-mail: rui.lopes@iscte.pt

R. J. Lopes

ISCTE - Instituto Universitário de Lisboa, Lisboa, Portugal

S. Jumisko-Pyykkö

Department of Pervasive Computing, Tampere University of Technology, Tampere, Finland e-mail: satu.jumisko-pyykko@tut.fi

H. -J. Zepernick

Blekinge Institute of Technology, Radio Communications Group, Karlskrona, Sweden

e-mail: hans-jurgen.zepernick@bth.se 
application scope" by Barkowsky et al. provides an introduction to this topic. Specifically, it presents essential aspects of current video quality metrics and their implementation in existing quality estimation schemes. Furthermore, the concept of hybrid video quality prediction and the Hybrid project of the Video Quality Experts Joint Effort Group are introduced. In "Audiovisual robustness: Exploring perceptual tolerance to asynchrony and quality distortion", Eg et al. investigate the interaction between subjective detection of temporal asynchrony of audio and video and media quality. Experiments to determine if the test persons can detect asynchrony in different audiovisual samples with different audio and video qualities have been carried out. The results show that for certain types of contents, the asynchrony detection is weakly influenced by the audiovisual quality.

Instrumenting applications and techniques for collecting UX metrics are chief tools in the development and support of QoE. The papers by Borowiak and Reiter, and Ickin et al. contribute to this topic. Borowiak and Reiter propose a novel methodology for rating degraded audiovisual (AV) content where users are able to freely adjust the quality of the stimulus once they notice the degradation, up to the point where the highest quality is reached. In "Quality Evaluation of Long Duration AV Content - An Extended Analysis using a Novel Assessment Methodology" they report their experiences made with this rating scheme when considering long duration AV content. The authors conclude that TV viewers are less sensitive to quality changes when they are in charge of the quality adjustment. Ickin et al. present in "VLQoE: Video QoE Instrumentation on the Smartphone" an instrumentation for QoE assessment on smart phones. An open source toolkit is provided that may open new approaches and research possibilities for investigators who are addressing the challenges of relating QoE understanding to device and software control. Since the tool is still at its infancy, the authors also suggest ways in which the toolkit could be improved. As a proof of concept, a specific investigation has been performed. The freeze duration and time between picture freezes for video transmission over the hypertext transfer protocol or the real-time streaming protocol to mobile devices is estimated and the user satisfaction is assessed by the means of a subjective study.

The two last papers of this issue study how QoE can be considered in the complete networked multimedia chain. The major claim of the paper entitled "Requirement Driven Prospects for Realizing User-Centric Network Orchestration" by Zinner et al. is that there is a need for adequate application-network application programming interface (API) that can support QoE. The authors state that smart applications should communicate directly with the network to maximize QoE. Therefore, a requirements based services orchestration methodology is implemented and tested with the aim of guaranteeing a satisfactory QoE to users. Castro et al. give in "Semantically Connected Web Resources with MPEG-21" an overview on the "MPEG-21 - Part 3: Digital Item Identification, Amendment 2: Digital item semantic relationships" standard. This paper explores how structured semantic descriptors and ontologies can be used to enhance the user experience while performing multimedia content semantic searches.

We would like to thank the Editor-in-Chief of the journal, Dr. Borko Furht for giving us the opportunity to present recent developments in the field of Multimedia QoE. We would like to thank the reviewers who have supported this issue and helped to increase the quality of its contributions and to all the authors who have contributed to this special issue by submitting their manuscripts. 
Acknowledgments We gratefully acknowledge grant CS11-009 from the Wiener Wissenschafts-, Forschungs-, und Technologiefonds (WWTF, Vienna Science and Technology Fund) to Ulrich Ansorge, Shelley Buchinger, and Otmar Scherzer.

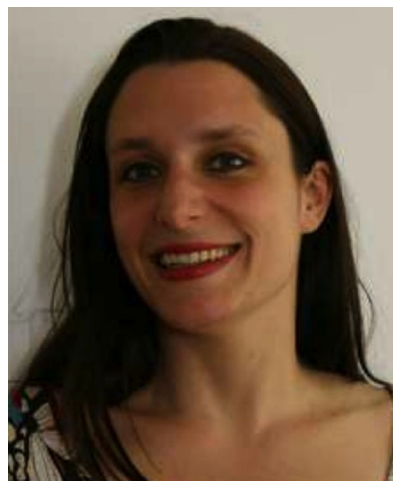

Shelley Buchinger received her M.Sc. degree in mathematics in 1999, in Education for Computer Science Management and Mathematics in 2008 and her Ph.D. (Dr. techn.) on Quality of Experience (QoE) for Mobile TV in February 2010, all three from the University of Vienna. In the frame of the Erasmus program she moved to Rome during her first master studies where she decided to stay for five years gaining experience in industry. Currently, she is engaged as post-doc researcher at the Cognitive Science research platform, University of Vienna where she is focusing her research on applications of visual attention models in medical imaging and ICT.

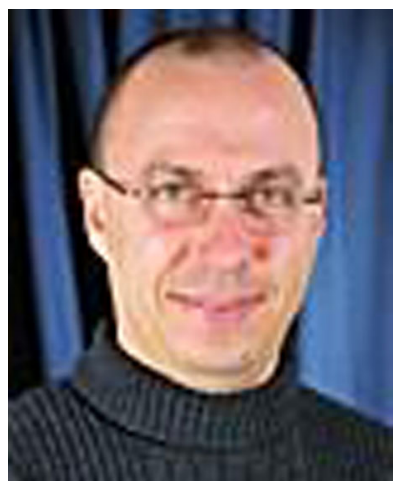

Rui J. Lopes received his undergraduate and M.Sc. degrees in Electrotechnical and Computer Engineering in 1993 an 1996 from Instituto Superior Técnico in Lisbon, Portugal and his Ph.D. in Computer Science from Lancaster University in Lancaster, U.K. He is a lecturer in the Information Science and Technology Department at ISCTE Lisbon University Institute in Lisbon, Portugal where he lectures Computer Networks and Telecommunication Services Engineering courses.His main research interests focus on QoE support and measurements in networked multimedia systems, and network related issues in complex systems. 


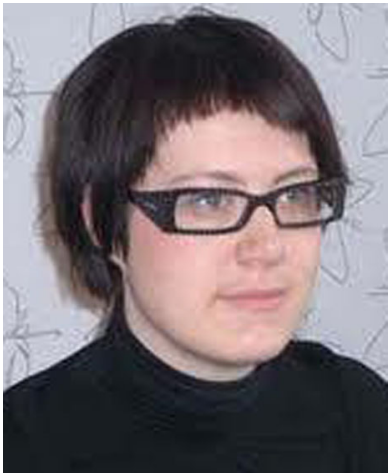

Satu Jumisko-Pyykkö received her M.Sc. degree in software engineering and Dr. (techn) degree in computer science from the Tampere University of Technology, Finland. Her $\mathrm{PhD}$ thesis focused on User-Centered Quality of Experience (UC-QoE) for mobile television and its evaluation methods. She is currently adjunct and assistant professor at the Tampere University of Technology and visiting post-doc researcher at Eindhoven University of Technology, Netherlands. Her research interests combine psychology with technology and are focused on experienced quality of novel audiovisual technologies, their evaluation methods especially in context of use, and future interaction technologies.

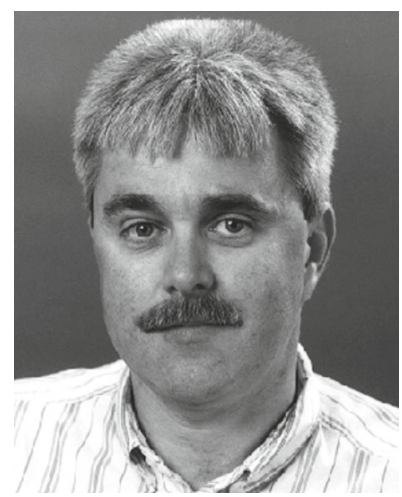

Hans-Jürgen Zepernick received the Dipl.-Ing. degree from the University of Siegen, Germany and the Dr.-Ing. degree from the University of Hagen, Germany, in 1987 and 1994, respectively. He was with Siemens AG, Munich, Germany, from 1987 to 1989 . He is currently a Professor of radio communications with the Blekinge Institute of Technology, Karlskrona, Sweden. Prior to this appointment, he held the positions of Professor of wireless communications at Curtin University of Technology, Bentley, Australia, Deputy Director of the Australian Telecommunications Research Institute, Perth, Australia, and Associate Director of the Australian Telecommunications Cooperative Research Centre. His current research interests include cooperative communications, cognitive radio networks, mobile multimedia, and perceptual quality assessment. 